\title{
Febrile neutropenic patients with solid neoplasms have few $P$. aeruginosa infections and higher antibacterial doses showed no benefit over lower doses
}

\author{
Roger A. Bitar* \\ Infectious Diseases Division, Kaiser Permanente, San Diego Medical Center, San Diego, CA, United States
}

Received: December 30, 2015

Accepted: February 22, 2016

Online Published: March 6, 2016

DOI: $10.5430 /$ jst.v6n1p94

URL: http://dx.doi.org/10.5430/jst.v6n1p94

\begin{abstract}
Background: Many febrile neutropenic patients (FNP) with solid tumors receive high dose antibacterial therapy adequate for $P$. aeruginosa infections. This study was designed to determine the frequency of $P$. aeruginosa infections in FNP with solid neoplasms and detect any differences in duration of fever, length of stay (LOS) and deaths of patients receiving low (LD) vs. high dose (HD) antibacterial therapy.

Methods: Electronic medical record databases were searched to identify patients with drug-induced neutropenia and fever. The resulting data was searched manually for patients with solid neoplasms and these charts were selected consecutively for manual review for additional inclusion criteria, comparison characteristics, such as type of neoplasm, comorbidities, potential risk factors such as duration of neutropenia, documented infections, and outcomes: duration of temperature to $<37.5^{\circ} \mathrm{C}$ and $<38^{\circ} \mathrm{C}$, length of stay (LOS), and cause of death. Components of the Multinational Association for Supportive Care of Cancer risk index (MASCCRI) were extracted and the index was calculated for each episode.

Results: The respective outcomes of LD vs. HD were: mean duration of temperature to $<38^{\circ} \mathrm{C}, 3$ vs. 3 days, and to $<37.5^{\circ} \mathrm{C}, 4$ vs. 4 days, mean LOS 6.3 vs. 6.6 days ( $p=.56$, TT; but $p<.01$, WRST), and LOS $\leq 10$ days, 90\% vs. 89\%, Zero vs. 2 developed P. aeruginosa infections.

Conclusions: LD antibacterials for FNP with solid neoplasms did not prolong the time to afebrile or the LOS, and only $2 P$. aeruginosa infections occurred. High dose antibacterial therapy may not be necessary for FNP with solid neoplasms.
\end{abstract}

Key Words: Fever, Neutropenia, Solid neoplasms, Antibacterials

\section{INTRODUCTION}

Cytotoxic chemotherapy for cancer frequently results in neutropenia and some of these neutropenic patients become febrile. In 1966 Bodey et al. identified depth and duration of neutropenia as risk factors for infection. If the absolute neutrophil count (ANC) was $<1,000 v s$. less than 100 , the incidence of infection was $14 \%$ or $\geq 24 \%$, respectively. ${ }^{[1]}$ For patients with solid neoplasms, the depth and duration of neutropenia is generally shorter than in patients receiving chemotherapy for those with hematoproliferative/lymphoproliferative malignancies. Figure 1 shows a comparison of the lengths of stay of patients with various malignancies following chemotherapy. Length of stay (LOS) is usually only a few days longer than the duration of neutropenia. In 1971 Schimpff et al. noted that P. aeruginosa had been "the most frequent cause of infection at the Baltimore

* Correspondence: Roger A. Bitar, M.D, MPH; Email: rhabit@yahoo.com; Address: Infectious Diseases Division, Retired, Kaiser Permanente, San Diego Medical Center, now with Mission Infectious Disease \& Infusion Consultants, Inc., Poway, CA, United States. 
Cancer Research Center during the recent years" and that $50 \%$ of the patients with pseudomonas bacteremia, identified between July 1968 and July 1969, died in 3 days. ${ }^{[2]}$ The number of patients with hematoproliferative/lymphoproliferative malignancies $v s$. solid neoplasms is not stated, but $P$. aeruginosa infections were more common in patients with "bone marrow" $v s$. other types. In a 1985 review of 410 episodes of $P$. aeruginosa bacteremia, early antibacterial therapy active $v s$. P. aeruginosa was crucial in reducing mortality, e.g. from $26 \%$ in the first 24 hours for those who received an antibacterial regimen inactive against $P$. aeruginosa to 5\% for those who received an active antibacterial regimen. ${ }^{[3]}$ Talcott et al. introduced the concept of stratification based on risk of complications in 1988, but did not specifically link stratification to type of malignancy or risk for infection due to $P$. aeruginosa, although patients with solid neoplasms were included. ${ }^{[4]}$ In a 1993 review article titled "Management of Fever in Patients with Cancer and Treatment-Induced Neutropenia," Pizzo referenced an article by Schimpff et al. "Origin of Infection in Acute Nonlymphocytic Leukemia" in support of $P$. aeruginosa as an important pathogen in neutropenic patients. ${ }^{[5]}$ The article by Schimpff obviously does not include patients with solid neoplasms. Although noting the decreasing frequency of $P$. aeruginosa infections in neutropenic patients and that patients with lymphomas and solid neoplasms have shorter duration of neutropenia, Pizzo emphasized the importance of using antibacterial therapy active against $P$. aeruginosa in febrile neutropenic patients (FNP). In 1997, the Infectious Disease Society of America published guidelines for the management of FNP, however, there is no stratification of patients for risk of infection based on type of malignancy, e.g. hematoproliferative/lymphoproliferative $v s$. solid neoplasms, or for $P$. aeruginosa infections. ${ }^{[6]}$ The recommended dose of Ceftazidime is $2 \mathrm{gm} \mathrm{q} 8 \mathrm{hrs}$, although this recommendation is followed by this statement "However, in some studies, lower doses of 1.0 gram every 8 hours have been used successfully in patients with solid tumors and with expected short periods of neutropenia." The Multinational Association for Supportive Care in Cancer risk index (MASCCRI) was introduced in 2000, purporting to be a methodology for stratification of neutropenic patients by risk of complications. ${ }^{[7]}$ (See critique of this methodology in reference 8). In this methodology, stratification is again not linked to type of neoplasm or risk for infection due to $P$. aeruginosa.

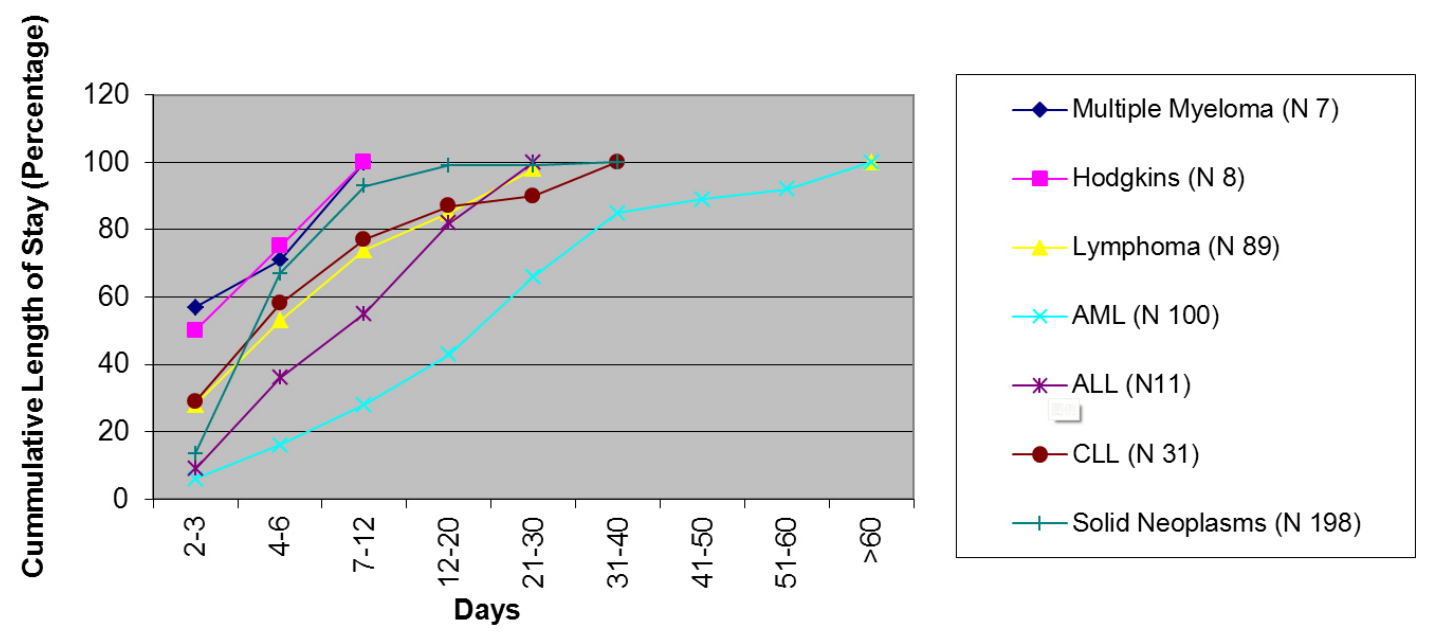

Figure 1. Cummulative length of stay in hospital vs. days in hospital for various type of malignancies post chemotherapy $(\mathrm{AML}=$ Acute myelocytic leukemia; $\mathrm{ALL}=$ Acute lymphoblastic leukemia; $\mathrm{CLL}=$ Chroni lymphocytic $)$.

The Infectious Disease Society of America (IDSA) updated the guidelines for treatment of FNP in 2002 and 2010, ${ }^{[9,10]}$ with risk stratification based on the MASCCRI, particularly emphasized in 2010. Although there has been some general stratification in studies of FNP with leukemia vs. solid neoplasm vs. lymphoma, risks for $P$. aeruginosa infections or poor outcomes were not provided, thus high-doses of antibacterial agents, adequate for $P$. aeruginosa infections, have been given to patients with solid neoplasms, as well as those with hematopoietic and lymphoproliferative malignancies. ${ }^{[11,12]}$ Other studies, however, have shown that patients with solid neoplasms are at lower risks for poor outcomes than those with hematopoietic and lymphoproliferative malignancies, likely due to less depth and duration of neutropenia. $^{[13]}$

It was my personal observation that patients with solid neoplasms, febrile when neutropenic, had less complications, recovered more quickly, and were less likely to have in- 
fections due to $P$. aeruginosa than FNP with hematopoietic/lymphoproliferative malignancies. I also concluded that they did not need high doses of antibacterial agents so I undertook this retrospective study to address two questions: Do patients treated with lower doses of antibacterial agents have longer duration of fever, longer LOS, and increased mortality compared to those receiving high dose antibacterials? How frequent are infections due to $P$. aeruginosa? Are $P$. aeruginosa infections frequent enough to justify high dose antibacterial therapy? Is there evidence demonstrating that low dose antibacterial therapy active against $P$. aeruginosa results in inadequate treatment?

\section{Patients And methods}

\subsection{Patients}

Inclusion criteria: inpatients with LOS > 24 hours, (note: it seemed that a stay of less than two days could not be attributed to the effectiveness of antibacterial therapy), $>18$ years old, with a solid neoplasm, who received chemotherapy, became neutropenic with an ANC of $<500$ cells/ $\mu$ - - a diagnosis on admission or discharge of neutropenic fever based on these codes-Drug induced neutropenia (288.0), and Fever presenting conditions classified elsewhere (780.61)-and who received antibacterial therapy for neutropenic fever. Patients were identified by searching electronic medical records of patients admitted consecutively to Kaiser Permanente San Diego Medical Center, from October 1, 2008 to November 15, 2010 and Kaiser Permanente Orange County Medical Center, and Kaiser Permanente Woodland Hills Medical Center from October 1, 2008 to April 30, 2010 for all inpatients with ICD9 codes 288.0 and 780.61. The charts of these patient episodes were consecutively selected and manually screened for inclusion criteria and data extracted, if inclusion criteria were met.

Data extracted, included: the type of solid neoplasm whether it was metastatic beyond local nodes, occurrence of fever associated with neutropenia, duration of fever, an ANC < 500 cells/ $\mu 1$, return of ANC to greater than 500 cells $/ \mu 1$, duration of neutropenia, age, gender, admission date, discharge date, LOS, reason for extended hospital stay, ICU care, comfort care, death, death date, reception of filgrastim, other diagnoses in the problem list which might be considered immunocompromising, smoking status (never, presently or former), serum creatinine $>2$, burden of illness, hypotension (SBP < 90), dehydration, outpatient or inpatient chemotherapy, types of infections, positive cultures, microorganisms, antimicrobial agents and doses, results of pertinent imaging studies. The other relevant parts of the chart were reviewed to determine the course of the patient in the hospital, the resolution of fever, and condition at end of hospitalization. The
Kaiser Permanente Southern California Institutional Review Board (IRB) approved the study.

\subsection{Antibacterial regimens}

The designation low dose (LD) or high dose (HD) is related to the usual dosing of the antibacterials with activity against aerobic, gram negative bacilli, particularly $P$. aeruginosa. LD antibacterial regimens were: Ceftazidime $1 \mathrm{gm}$ q8 hrs, Cefepime 1 gm q 12 hrs, Aztreonam 1 gm q 8 hrs, Piperacillin/tazobactam 3.375 q6 hrs, Ceftriaxone 1-2 gm q 24 hrs, Ciprofloxacin $400 \mathrm{mg} \mathrm{q} 12 \mathrm{hrs}$ or $500 \mathrm{mg}-750 \mathrm{mg}$ po q 12 hrs, Imipenem $500 \mathrm{mg} \mathrm{q} 8 \mathrm{hrs}$, Ertapenem $1 \mathrm{gm} \mathrm{q}$ $24 \mathrm{hrs}$, Levofloxacin $500 \mathrm{mg}$ po q $24 \mathrm{hrs}$. HD antibacterial regimens were: Ceftazidime 2 gm q8 hrs, Cefepime 2 gm q 12 hrs, Aztreonam 2 gm q8 hrs, Piperacillin/tazobactam 4.5 mg q 4-6 hrs. No patient received Ciprofloxacin $400 \mathrm{mg}$ IV or $750 \mathrm{mg}$ or $500 \mathrm{mg}$ po, q8 hrs. In both groups vancomycin and gentamicin were per pharmacy protocol, metronidazole $500 \mathrm{mg}$ q 8-12 hrs po or IV, Clindamycin $600 \mathrm{mg}$ IV q 8 hrs, Ampicillin 2 gm q 4-6 hrs.

\subsection{Filgrastim}

Patients receiving filgrastim were identified and a chart review was conducted to verify the electronic data extraction for those identified as not receiving filgrastim.

\subsection{Study design}

Retrospective review of electronic medical records as noted above.

\subsection{Definitions}

Febrile neutropenia was defined based on accepting the admitting diagnosis recorded by the admitting physician and an ANC of $<500$ cells/ $\mu 1$, except for two occurrences when it was between 500 and 600 cells $/ \mu 1$. Some patients received chemotherapy as inpatients and developed neutropenic fever. In these inpatients, the ANC had to be $<500$ cells $/ \mu$ l and the temperature greater than or equal to $38^{\circ} \mathrm{C}$ to meet criteria for neutropenic fever. The duration of fever was the number of days elapsed from the calendar day, defined as midnight to midnight, of admission to the calendar day of the last temperature to reach $38^{\circ} \mathrm{C}$ or $37.5^{\circ} \mathrm{C}$. The number of days from admission to the first day the temperature was less than $38^{\circ} \mathrm{C}$ or $37.5^{\circ} \mathrm{C}$, and never again reached $38^{\circ} \mathrm{C}$ or $37.5^{\circ} \mathrm{C}$, was the number of days to afebrile 38 and similarly to afebrile 37.5 . Response to therapy was designated as afebrile 38 or afebrile 37.5. However, there are patients in this study who had artificially prolonged duration of fever due to a subsequent "blip" in temperature above $37.5^{\circ} \mathrm{C}$ despite having been afebrile for two or more days. LOS in hospital was calculated by subtracting the admission date from the discharge date and 
adding on $\mathrm{e}^{[1]}$ for the data in Figures 1 and 2, so any part of a day counts as one day.

Table 1. Patient characteristics

\begin{tabular}{|c|c|c|}
\hline & $\begin{array}{l}\text { Low, } \\
\mathrm{n}=\mathbf{1 0 0}\end{array}$ & $\begin{array}{l}\text { High, } \\
\text { n=98 }\end{array}$ \\
\hline \multicolumn{3}{|l|}{ Age } \\
\hline Range & $33-86$ & $18-85$ \\
\hline Median & 61 & 60.5 \\
\hline \multicolumn{3}{|l|}{ Gender } \\
\hline M & 30 & 27 \\
\hline $\mathrm{F}$ & 70 & 71 \\
\hline \multicolumn{3}{|l|}{ Co-morbidities } \\
\hline Diabetes & 8 & 11 \\
\hline CKD III or IV & 9 & 9 \\
\hline Cirrhosis & 1 & 0 \\
\hline Prednisone & 1 & 0 \\
\hline Rheumatoid Arthritis & 0 & 2 \\
\hline CREST & 0 & 1 \\
\hline PMR & 1 & 1 \\
\hline Organ Transplant & 1 & 0 \\
\hline Anti-TNF & 1 & 0 \\
\hline Hepatitis B & 1 & 0 \\
\hline Hepatitis C & 3 & 2 \\
\hline HIV & 0 & 2 \\
\hline Hypogammaglobulinemia & 1 & 1 \\
\hline Hemochromatosis & 0 & 2 \\
\hline Ulcerative Colitis & 0 & 1 \\
\hline \multicolumn{3}{|l|}{ Smoking } \\
\hline never & 58 & 62 \\
\hline former & 38 & 31 \\
\hline present & 4 & 5 \\
\hline Metastases beyond local nodes & 33 & 39 \\
\hline MASCC score & 22.98 & 22.87 \\
\hline Filgrastim & $84 \%$ & $94.9 \%$ \\
\hline $\begin{array}{l}\text { Chemotherapy density and intensity } \\
\text { meeting GCSF criteria }\end{array}$ & $27.6 \%$ & $24 \%$ \\
\hline \multicolumn{3}{|l|}{ Malignancies } \\
\hline Head \& Neck & 3 & 6 \\
\hline Breast & 50 & 43 \\
\hline Lung & 7 & 11 \\
\hline GI & 21 & 18 \\
\hline Uterus & 1 & 0 \\
\hline Ovary & 4 & 4 \\
\hline Prostate & 5 & 2 \\
\hline Bladder & 2 & 2 \\
\hline Testis & 2 & 0 \\
\hline Sarcoma & 1 & 11 \\
\hline Melanoma & 0 & 1 \\
\hline Neuroectodermal & 2 & 0 \\
\hline Unknown & 2 & 0 \\
\hline
\end{tabular}

Note. CKD = chronic kidney disease; CREST = Calcinosis, Raynaud phenomenon, Esophageal dysmotility, sclerodactyly, and Telangiectasia; PMR $=$ Polymyalgia rheumatica; Anti-TNF = Tumor necrosis factor inhibitor; GCSF = granulocyte colony stimulating factor. As an age-range grouped variable, the $t$-test between the dose groups had significance, $p=.05$ (TT), while age as a continuous variable, the significance was $p=.04$ (TT). There was a tendency for the LD to be younger.
Types of infections described by the physicians of record were accepted, with these conditions. Any patient with at least one positive blood culture was defined as being bacteremic, excluding those patients who had only one blood culture positive for a typical contaminant from the skin, e.g. coagulase negative staphylococcus (CNS), non-JK diphtheroids. Pneumonia was based on the official chest X-ray report. Urinary tract infection (UTI) was defined as $>10^{5}$ colony forming unit/milliliter. Diverticulitis was defined as a patient with abdominal pain and diverticula on CT scan, in addition to one or more of these findings: pericolic infiltration of fatty tissue, colonic wall thickening or pericolonic abscess. Colitis was diagnosed based on a CT scan of the abdomen demonstrating colonic wall thickening. MASCCRI was calculated based on published criteria. ${ }^{[7]}$ Each patient entry represents a unique admission to the hospital and is called a patient episode. There were three patients in the LD and five patients in the HD, which were admitted twice and received LD or HD both times, five patients in each group that were admitted twice and received LD during one admission and HD during the other admission. There was one patient admitted three times, each time HD was given. All patients had completely recovered from the prior episode of fever and neutropenia by the time a subsequent admission to the hospital met inclusion criteria. Treatment was considered effective if there was resolution of fever and other signs of infection prior to discharge from the hospital. Death or failure to control infection was considered a failure of treatment.

\subsection{Statistical analysis}

A less than $10 \%$ difference in outcomes between the groups was not considered clinically relevant. To show that the two groups could be considered equivalent, with 100 per group (LD vs. HD) the difference would need to be less than 0.55 days, with a common standard deviation of 1.5 days using a $t$-test. At this level there would be more than $80 \%$ power to declare the nonequivalence statistically significant at the 0.05 level. Since the intent is to show no statistically significant difference between the groups, the more statistically significant value from either the $t$-test (TT), Wilcoxon rank sum test (WRST), or the Pearson chi-square test (PCST), would be chosen.

\section{Results}

\subsection{Patient characteristics}

During the time periods mentioned, 100 patient episodes in the LD and 98 patient episodes in the HD met inclusion criteria. Characteristics of the LD and the HD were similar (see Table 1), except there was a tendency for the LD to be slightly younger (See commentary below Table 1) and for the overrepresentation of sarcomas in the HD (LD 1 vs. 
HD 11, reason unknown). There was no difference between the mean MASCCRI ${ }^{[7]}$ of the LD vs. the HD. Eighty- four percent of the LD and $94.9 \%$ of the HD received filgrastim.

\subsection{Types of infections and microbiology}

As shown in Table 2, if colitis and enteritis are excluded, there were 26 clinically documented infections in the LD (100 patient episodes) and 30 in the HD (98 patient episodes).

Table 2. Infections and microbiology, low-dose vs. high-dose

\begin{tabular}{|c|c|}
\hline Low dose-Number 36 & High dose-Number 35 \\
\hline Pneumonia & Pneumonia \\
\hline Bacteremia, fusobacterium & UTI, enterococcus \\
\hline Pneumonia, M. avium-intracellulare & PICC, Cellulitis \\
\hline UTI, enterococcus & Bacteremia, S. aureus; Pneumonia, S. aureus, P. aeruginosa; UTI, VRE \\
\hline Colitis* & Bacteremia, S. bovis; UTI, E. Coli \\
\hline $\begin{array}{l}\text { Bacteremia, S. pneumoniae, viridans streptococcus; } \\
\text { Pnuemonia; Intestinal obstruction; UTI, K. Oxytoca }\end{array}$ & Bacteremia, $P$. aeruginosa; UTI, enterococcus \\
\hline $\begin{array}{l}\text { Bacteremia, E. Coli, K. pneumoniae, viridans streptococcus; } \\
\text { Decubitus ulcer; UTI, E. Coli }\end{array}$ & Bacteremia viridans streptococcus; C. difficile Colitis \\
\hline Bacteremia, E. Coli; UTI, E. Coli & Bacteremia, Grp G Streptococcus \\
\hline Bacteremia, enterococcus; Intestinal obstruction; Enteritis* & Bacteremia, E. Coli \\
\hline Bacteremia, S. aureus; PICC & Bacteremia, Chryseomonas \\
\hline Pneumonia; C. difficile Colitis & Bacteremia, S. aureus, viridans streptococcus, E. Coli \\
\hline Pneumonia & Bacteremia, E. Coli \\
\hline Pneumonia & UTI, E. Coli \\
\hline UTI, E. Coli & Pneumonia \\
\hline UTI, E. Coli & Pneumonia \\
\hline UTI, E. Coli & UTI, E. Coli, Grp B streptococcus \\
\hline UTI, K. oxytoca & Colitis; UTI, E. Coli \\
\hline C. difficile collitis & UTI, enterococcus \\
\hline Colitis & UTI, K. pneumoniae \\
\hline Colitis & C. difficile Colitis \\
\hline Colitis & C. difficile Colitis \\
\hline Colitis & C. difficile Colitis \\
\hline Colitis & Colitis \\
\hline Colitis & Colitis \\
\hline Colitis & Colitis \\
\hline Colitis & Colitis \\
\hline Enteritis & Colitis \\
\hline Intestinal obstruction; Infected ascites, C. albicans & Intestinal Obstruction \\
\hline Perirectal absces, S. aureus, B. fragilis & Cellulitis; Sinusitis \\
\hline Finger abscess & Cellulitis \\
\hline Cellulitis & Cellulitis \\
\hline Cellulitis & Sinusitis \\
\hline Cellulitis & Diverticultis \\
\hline PICC, Coagulase negative staphylococcus & Cellulitis \\
\hline Diverticulitis & Diverticultis \\
\hline Diverticulitis & \\
\hline
\end{tabular}

Note. Colitis and enteritis may not be infectious but chemotherapy induced; PICC = percutaneously inserted central catheter; UTI = urinary tract infection; VRE = vancomycin resistant enterococcus.

Bacteremia was documented in six patients in the LD and 9 in the HD. The isolates and sources shown in Table 1 are typical for hospitalized patients including two polymicrobial bacteremias in the LD group and one in the HD group. Eight patients in the LD and nine in the HD had documented UTI with common urinary tract isolates. Two patients in the HD group had $P$. aeruginosa isolated, one from the blood and one from the sputum.

Table 2 also shows a total tally of infections and microorganisms isolated. Chi-square tests of comparisons for each type of infection failed to reject the null hypothesis of no 
difference between the groups (direct comparison in table scess and was readmitted non-neutropenic within seven days not shown). One patient in the HD was discharged non- of discharge when discovered, thus not meeting inclusion neutropenic and afebrile with an unrecognized perirectal ab- criteria for this subsequent admission.

Table 3. Antibacterial combinations administered to patients with solid neoplasms

\begin{tabular}{|c|c|c|}
\hline Antimicrobial regimens & Low dose & High dose \\
\hline Ceftazdime, Ciprofloxacin/Levofloxacin & 20 & 24 \\
\hline Ceftazdime, Ciprofloxacin/Levofloxacin, Vancomycin & 11 & 11 \\
\hline Ceftazdime, Ciprofloxacin/Levofloxacin, Vancomycin, Metronidazole & 2 & 4 \\
\hline Ceftazdime, Ciprofloxacin/Levofloxacin, Metronidazole & 7 & 2 \\
\hline Ciprofloxacin/Levofloxacin, Vancomycin & & 1 \\
\hline Cefepime, Ciprofloxacin, Metronidazole & & 1 \\
\hline Ceftazidime, Vancomycin & 5 & 17 \\
\hline Cefepime, Vancomycin & & 1 \\
\hline Piperacillin/Tazobactam, Ciprofloxacin, Vancomycin, Metronidazole & & 1 \\
\hline Ceftazdime & 10 & 12 \\
\hline Ceftazdime, Vancomycin, Metronidazole & & 5 \\
\hline Cefepime, Ciprofloxacin, Vancomycin & & 1 \\
\hline Ceftazidime, Vancomycin, Metronidazole & & 4 \\
\hline Ceftazidime, Metronidazole & & 5 \\
\hline Cefepime, Ciprofloxacin, Vancomycin, Metronidazole & & 1 \\
\hline Cefepime & & 2 \\
\hline Aztreonam, Clindamycin & & 1 \\
\hline Ceftazidime, Vancomycin, Clindamycin & & 1 \\
\hline Cefepime, Vancomycin & & 1 \\
\hline Ceftazidime, Vancomycin, Gentimicin & 1 & 1 \\
\hline Ceftazdime, Ciprofloxacin/Levofloxacin, Gentamicin, Metronidazole & & 1 \\
\hline Piperacillin/Tazobactam, Ciprofloxacin & 2 & 1 \\
\hline Cprofloxacin & 7 & \\
\hline Ceftazdime, Ciprofloxacin/Levofloxacin, Clindamycin & 2 & \\
\hline Ciprofloxacin, Vancomycin, Metronidazole & 1 & \\
\hline Ceftazdime, Ciprofloxacin/Levofloxacin, Vancomycin, Ampicillin & 1 & \\
\hline Amoxicillin/clavulanate, Gentamicin & 1 & \\
\hline Gentamicin, Clindamycin & 1 & \\
\hline Aztreonam, Ciprofloxacin & 1 & \\
\hline Aztreonam, Ciprofloxacin, Vancomycin & 1 & \\
\hline Piperacillin/Tazobactam & 8 & \\
\hline Piperacillin/Tazobactam, Ciprofloxacin, Vancomycin, Metronidazole & 1 & \\
\hline Ceftazidime, Piperacillin/tazobactam & 1 & \\
\hline Piperacillin/Tazobactam, Vancomycin & 6 & \\
\hline Piperacillin/Tazobactam, Ciprofloxacin, Vancomycin & 2 & \\
\hline Moxifloxacin & 2 & \\
\hline Ceftriaxone & 1 & \\
\hline Ciprofloxacin, Metronidazole & 1 & \\
\hline Vancomycin & 1 & \\
\hline Ceftriaxone, Vancomycin & 1 & \\
\hline Cefotaxime, Vancomycin, Metrondazole & 1 & \\
\hline Ertapenem, Linezolid & 1 & \\
\hline Imipenem (500mg every 8 hours, Creatinine 0.8) & 1 & \\
\hline Total & 100 & 98 \\
\hline
\end{tabular}




\subsection{Antibacterial agents}

For 198 patient episodes, 43 different initial antibacterial regimens were administered (see Table 3 ). The most frequent regimen in both the LD and the HD was the combination of ceftazidime and ciprofloxacin. The most frequently administered single drug in any regimen was ceftazidime, followed by ciprofloxacin. In the $\mathrm{LD}$, the initial regimen was changed 12 times, but analysis by clinical criteria, suggests that only four may have benefited from the change. The initial regimen was changed 13 times in the HD, and only three may have benefited. One patient received only vancomycin, one patient received only amoxicillin/clavulanate, and one patient received only amoxicillin/clavulanate and clindamycin.

\subsection{Response to therapy}

\subsubsection{Length of stay}

Figure 2 shows the distribution of the LOS of the two groups. The mean LOS was 6.3 days (range 3-31) for the LD compared to 6.6 days (range 3-19) for the HD. While these averages seem similar, the distributions seem to be different $(p=$ .62 , TT; but $p<.01$, WRST).

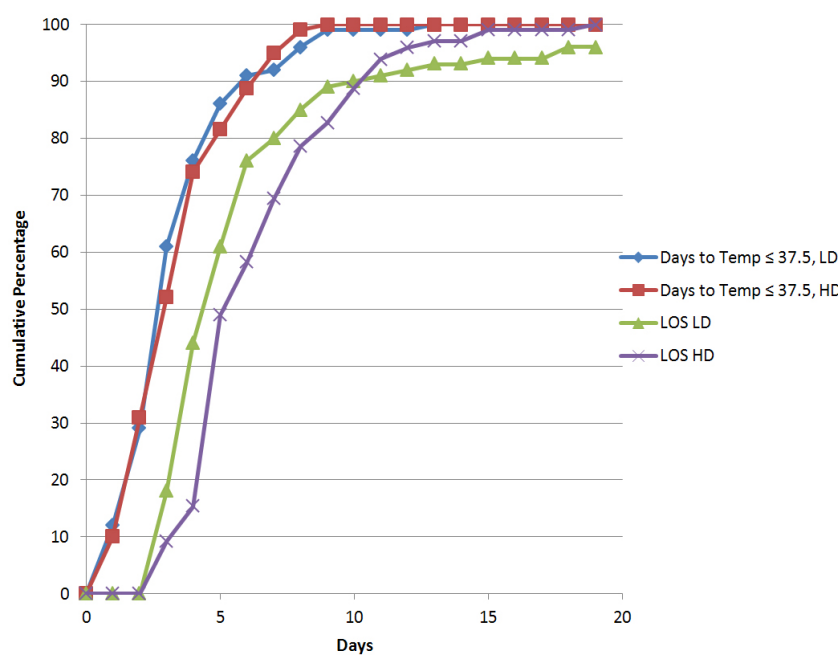

Figure 2. Cumulative percentage: Low vs. high dose antibacterials for time to temperature $<37.5$ and length of stay

\subsubsection{Duration of neutropenia}

The mean duration of neutropenia in the LD was 2.5 days (range 1-9) vs. 2.96 days (range 1-9) for the $\operatorname{HD}(p=.02$, WRST).

\subsubsection{Duration of fever}

The mean duration of fever in the LD was 3 (range 1-12) days to $<38^{\circ} \mathrm{C}$ vs. 3 (range 1-8) days for the HD and 4 (range 1-13) days to $<37.5^{\circ} \mathrm{C}$ in the LD vs. 4 (range 1-9) days in the HD. See Figure 2 for cumulative percentages.

\subsubsection{Deaths}

In the LD, 7 of 100 patients died in the hospital, but only two of the deaths were related to the infection present when the patient was admitted for FNP. In the HD, 4 of 98 patients died, but only 3 of 4 , who died in the hospital, died due to the infection present when admitted.

\section{Discussion}

The combined data from the LD and the HD groups demonstrate that patients with solid neoplasms have brief episodes of neutropenia (mean less than three days), infections that are controlled promptly (afebrile within four days of admission), and relatively short inpatient stays (89\% discharged in 10 days). They seldom die of the infections responsible for hospitalization (2\%-4\%); and infrequently develop P. aeruginoas infections (1\%).

\subsection{Goals of study addressed}

Do patients treated with lower doses of antibacterial agents have longer duration of fever, longer lengths of stay (LOS), and increased mortality compared to those receiving high dose antibacterials? There were no differences in the time required to become afebrile, the duration of hospitalization, or mortality from initial infections in the 2 groups. The duration of fever in this study is similar to the duration recorded by Elting et al. in a previous study of patients with solid neoplasms. ${ }^{[14]}$ The number of deaths attributed to the initial infection in this study of LD and HD treatment groups (2/100 vs. 3/ 98) is also concordant with other reports which have shown that FNP with solid neoplasms have less frequent fatal infectious complications than patients with acute leukemia. ${ }^{[15]}$

How frequent are infections due to $P$. aeruginosa? Infrequent, 2/198.

Are $P$. aeruginosa infections frequent enough in FNP to justify high dose antibacterial therapy? The answer is a matter of opinion since $P$. aeruginosa infections are infrequent. However, there is other data which is pertinent to this question. P. aeruginosa is infrequently isolated at the San Diego Medical Center (only 26 isolates, or 1.2 per month between March 2, 2008 and December 31, 2009). Furthermore, at least eight studies have found a low frequency of $P$. aeruginosa infections in FNP with solid neoplasms and patients at lower risk for complications. ${ }^{[16-23]}$ The cumulative number of episodes of 'febrile neutropenia' in these studies was 1,117 , with four episodes of $P$. aeruginosa bacteremia. In the studies reporting urine cultures, only 2 of 885 with urine cultures were positive for $P$. aeruginosa. No deaths related to $P$. aeruginosa, were reported in these studies. When the data from the present study are added to these studies, there are 
a total of 1,315 episodes with blood cultures, five positive for $P$. aeruginosa $(0.38 \%)$ and a total of 1,083 episodes with urine cultures, two positive for $P$. aeruginosa $(0.18 \%)$. Of these 1,315 patients, there was one death not likely due to $P$. aeruginosa, which was isolated from a sputum culture.

Is there evidence demonstrating that low dose antibacterial therapy active against $P$. aeruginosa results in inadequate treatment? Not in this study because there were only 2 patients with $P$. aeruginosa infections. However, there is literature which supports the efficacy of low dose anti-pseudomonal antibacterial agents. In 1994, a randomized trial of Ceftazidime $1 \mathrm{gm} \mathrm{q} 8 \mathrm{hrs}$ vs. Ceftazidime 2 gm q8 hrs, each coupled with a single daily dose of Tobramycin, was conducted in 150 patients with hematoproliferative/lymphoproliferative malignancies, lymphomas, bone marrow aplasia/dysplasias, and bone marrow transplants. At $96 \mathrm{hrs}$, no statistical difference was identified in overall response rates of $70 \%$ vs. $60 \%$, respectively, with complete response rates of $32 \%$ vs. $27 \%$, respectively. ${ }^{[24]}$

In their study of $P$. aeruginosa bacteremia in cancer patients, Chatzinikolaou, et al. ${ }^{[25]}$ made a number of important observations that support the findings at the Kaiser Permanente medical centers. Among the most pertinent were that the frequency of $P$. aeruginosa bacteremia decreased in patients with solid neoplasms between 1985 and 2000: at their institution, $82 \%$ of solid neoplasm patients with $P$. aeruginosa bacteremia were cured, and 6 of the 7 patients who received only ciprofloxacin recovered. They also reiterated that the outcome of infections during neutropenia was related to the recovery of the neutrophil count and found that $97 \%$ of patients whose neutrophil count increased above 100 cells $/ \mu 1$ were cured.

\subsection{Limitations of this study}

First, this was a retrospective study by one physicianinvestigator, however, one with 29 years as an infectious disease physician and 44 years as a physician. Second, patients were members of Kaiser Health Plan in southern California and were admitted to 3 Kaiser Permanente Hospitals, so most would have been employed at the time of admission, or had been employed, to be able to afford health plan dues, so they might not be representative of the general population or of patients managed in other institutions. However, demographics of members of Kaiser Permanente San Diego, studied in October, November, and December 2009 , were very similar to those of the population of the county of San Diego (http: //wwwnc.cdc.gov/eid/arti cle/22/2/15-0618-techapp1.pdf). Although Table 1 shows that the per cent of patients meeting the criteria for granulocyte colony stimulating factor was similar, it is possible that other institutions may use more potent chemotherapy: for patients with solid neoplasms, and the risk of infections, in general, and infections due to $P$. aeruginosa is greater. This was a study of FNP with solid neoplasms who have in the past received less potent cytotoxic chemotherapy than patients with hematoproliferative/lymphoproliferative malignancies. The depth and duration of the neutropenia in the latter patients is usually greater and longer than those receiving chemotherapy for solid neoplasms and, thus, are at greater risk for infections, possibly including $P$. aeruginosa. Thus, one should not extrapolate the findings in this study to patients receiving chemotherapy for hematoproliferative/lymphoproliferative malignancies.

In summary, this data supports the use of low dose antibacterial therapy for FNP with solid neoplasms for three reasons. 1) Patients treated with LD vs. HD antibacterials did not have longer duration of fever 2) or longer LOS. 3) P. aeruginosa is an infrequent pathogen in FNP with solid neoplasms. A prospective study investigating lower dose antibacterial therapy for FNP with solid neoplasms would be useful.

\section{CONFlicts of InTEREST Disclosure}

The author declares that there is no conflict of interest statement.

\section{REFERENCES}

[1] Bodey GP, Buckley M, Sathe YS, et al. Quantitative relationships between circulating leukocytes and infection in patients with acute leukemia. Ann Intern Med. 1966 Feb; 64(2): 328-40. PMid:5216294. http://dx.doi.org/10.7326/0003-4819-64-2-328

[2] Schimpff S, Satterlee W, Young VM, et al. Empiric therapy with carbenicillin and gentamicin for febrile patients with cancer and granulocytopenia. N Engl J Med. 1971 May 13; 284(19): 1061-5. PMid:4994878. http://dx.doi.org/10.1056/NEJM197105132 841904

[3] Bodey GP, Jadeja L, Elting L. Pseudomonas bacteremia. Retrospective analysis of 410 episodes. Arch Intern Med. 1985 Sep; 145(9):
1621-9. PMid:3927867. http://dx.doi.org/10.1001/archint e.1985.00360090089015

[4] Talcott JA, Finberg R, Mayer RJ, et al. The medical course of cancer patients with fever and neutropenia. Clinical identification of a low-risk subgroup at presentation. Arch Intern Med. 1988 Dec; 148(12): 2561-8. PMid:3196123. http://dx.doi.org/10.1001 /archinte.1988.00380120031007

[5] Pizzo PA. Management of fever in patients with cancer and treatmentinduced neutropenia. N Engl J Med. 1993 May 6; 328(18): 1323-32. PMid:8469254. http://dx.doi.org/10.1056/NEJM199305063 281808

[6] Hughes WT, Armstrong D, Bodey GP, et al. 1997 guidelines for the 
use of antimicrobial agents in neutropenic patients with unexplained fever. Infectious Diseases Society of America. Clin Infect Dis. 1997 Sep; 25(3): 551-73. PMid:9314442. http://dx.doi.org/10.10 $86 / 513764$

[7] Klastersky J, Paesmans M, Rubenstein EB, et al. The Multinational Association for Supportive Care in Cancer risk index: A multinational scoring system for identifying low-risk febrile neutropenic cancer patients. J Clin Oncol. 2000 Aug; 18(16): 3038-51. PMid:10944139.

[8] Bitar RA. Utility of the Multinational Association for Supportive Care in Cancer (MASCC) Risk Index Score as a Criterion for Nonadmission in Febrile Neutropenic Patients with Solid Tumors. Perm J. 2015 Summer; 19(3): 37-47. http://dx.doi.org/10.7812/tpp /14-188

[9] Hughes WT, Armstrong D, Bodey GP, et al. 2002 guidelines for the use of antimicrobial agents in neutropenic patients with cancer. Clin Infect Dis. 2002 Mar 15; 34(6): 730-51. PMid:11850858. http://dx.doi.org/10.1086/339215

[10] Freifeld AG, Bow EJ, Sepkowitz KA, et al. Infectious Diseases Society of America. Clinical practice guideline for the use of antimicrobial agents in neutropenic patients with cancer: 2010 update by the infectious diseases society of america. Clin Infect Dis. 2011 Feb 15; 52(4): e56-93. PMid:21258094. http://dx.doi.org/10.10 93/cid/cir073

[11] Peacock JE, Herrington DA, Wade JC, et al. Ciprofloxacin plus piperacillin compared with tobramycin plus piperacillin as empirical therapy in febrile neutropenic patients. A randomized, double-blind trial. Ann Intern Med. 2002 Jul 16; 137(2): 77-87. PMid:12118962. http://dx.doi.org/10.7326/0003-4819-1 37-2-200207160-00005

[12] Pizzo PA, Hathorn JW, Hiemenz J, et al. A randomized trial comparing ceftazidime alone with combination antibiotic therapy in cancer patients with fever and neutropenia. N Engl J Med. 1986 Aug 28; 315(9): 552-8. PMid:3526155. http://dx.doi.org/10.1056/N EJM198608283150905

[13] Rolston KV. New trends in patient management: risk-based therapy for febrile patients with neutropenia. Clin Infect Dis. $1999 \mathrm{Sep} ; 29(3)$ : 515-21. PMid:10530438. http://dx.doi.org/10.1086/598624

[14] Elting LS, Lu C, Escalante CP, et al. Outcomes and cost of outpatient or inpatient management of 712 patients with febrile neutropenia. J Clin Oncol. 2008 Feb 1; 26(4): 606-11. PMid:18235119. http://dx.doi.org/10.1200/JC0.2007.13.8222

[15] DePauw B, Deresinski SC, Feld R, et al. Ceftazidime compared with piperacillin and tobramycin for the empiric treatment of fever in neutropenic patients with cancer. A multicenter randomized trial. The Intercontinental Antimicrobial Study Group. Ann Intern Med. 1994 120(10): 834-44. http://dx.doi.org/10.7326/0003-4819-1 20-10-199405150-00004
[16] Chamorey E, Magne N, Foa C, et al. Ceftriaxone monotherapy for the treatment of febrile neutropenia in patients with solid tumors: a prospective study of 100 episodes. Med Sci Monit. 2004 Nov; 10(11): 119-25. PMid:15507863.

[17] Escalante CP, Weiser MA, Manzullo E, et al. Outcomes of treatment pathways in outpatient treatment of low risk febrile neutropenic cancer patients. Support Care Cancer. 2004 Sep; 12(9): 657-62. PMid:15185134.

[18] Freifeld A, Marchigiani D, Walsh T, et al. A double-blind comparison of empirical oral and intravenous antibiotic therapy for low-risk febrile patients with neutropenia during cancer chemotherapy. N Engl J Med. 1999 Jul 29; 341(5): 305-11. PMid:10423464. http://dx.doi.org/10.1056/NEJM199907293410501

[19] Hidalgo M, Hornedo J, Lumbreras C, et al. Outpatient therapy with oral ofloxacin for patients with low risk neutropenia and fever: a prospective, randomized clinical trial. Cancer. 1999 Jan 1; 85(1): 213-9. http://dx.doi.org/10.1002/(SICI) 1097-0 142(19990101)85:1<213: :AID-CNCR29>3.0.CO;2-D

[20] Innes H, Smith D, O'Reilly S, et al. Oral antibiotics with early hospital discharge compared with in-patient intravenous antibiotics for low-risk febrile neutropenia in patients with cancer: a prospective randomised controlled single centre study. Br J Cancer. 2003; 89(1): 43-9. PMid:12838298. http://dx.doi.org/10.1038/s j . bjc. 6600993

[21] Malik IA, Khan WA, Karim M, et al. Feasibility of outpatient management of fever in cancer patients with low-risk neutropenia: results of a prospective randomized trial. Am J Med. 1995 Mar; 98(3): 224-31. http://dx.doi.org/10.1016/S0002-9343(99)80367-2

[22] Niho S, Ohe Y, Goto K, et al. Randomized trial of oral versus intravenous antibiotics in low-risk febrile neutropenic patients with lung cancer. Jpn J Clin Oncol. 2004 Feb; 34(2): 69-73. PMid:15067098. http://dx.doi.org/10.1093/jjco/hyh019

[23] Sebban C, Dussart S, Fuhrmann C, et al. Oral moxifloxacin or intravenous ceftriaxone for the treatment of low-risk neutropenic fever in cancer patients suitable for early hospital discharge. Support Care Cancer. 2008 Sep; 16(9): 1017-23. PMid:18197434. http://dx.doi.org/10.1007/s00520-007-0383-z

[24] Gibson J, Johnson L, Snowdon L, et al. A randomised dosage study of ceftazidime with single daily tobramycin for the empirical management of febrile neutropenia in patients with hematological diseases. Int J Hematol. 1994 Aug; 60(2): 119-27. PMid:7948961.

[25] Chatzinikolaou I, Abi-Said D, Bodey GP, et al. Recent experience with Pseudomonas aeruginosa bacteremia in patients with cancer: Retrospective analysis of 245 episodes. Arch Intern Med. $2000 \mathrm{Feb}$ 28; 160(4): 501-9. PMid:10695690. http://dx.doi.org/10.10 $01 /$ archinte.160.4.501 